\title{
A Study on Problems in Facilitation of Urban and Rural Integration and Countermeasures
}

\author{
DongXin Wang ${ }^{1, a^{*}}$, and DongYang Geng ${ }^{2, b}$ \\ ${ }^{1}$ Instructor, Shijiazhuang University of Economics, Shijiazhuang, 050031, China. \\ ${ }^{2}$ Instructor, Shijiazhuang University of Economics,Shijiazhuang, 050031,China. \\ *a371020915@qq.com, ${ }^{\mathrm{b}}$ 15200012621@139.com
}

Keywords: urban and rural integration, urbanization, urban and rural dual structure

Abstract. The urban-rural dual structure is a product at a certain stage in China's economic and social development, which causes the inequality and imbalance of economic and social development in rural and urban areas as well as severely restricts the construction of a holistic well-being society. It is inevitable and primary to break the urban-rural dual structure and facilitate the common prosperity of rural and urban areas in the construction of modernization. In terms of the facilitation of urban and rural integration, the paper analyzes the problems and provides some countermeasures from the perspective of enhancing cadres' ideological education, strengthening system reforms, perfecting employment service system and accelerating the establishment of agricultural industrialization mechanism.

\section{Reasons for the problems in urban and rural integration}

Some people are not active ideologically and are restricted by the previous system

As the main subject that exerts urban and rural integration, some people do not have a good understanding of the policies. With rigid ideas, they are not willing to change the current situation or accept new things. They take a negative attitude. Moreover, due to the low education and weak working ability, they think that doing farming work can bring benefits ${ }^{[1]}$. On the contrary, they think that it is hard to seek jobs in cities. They would rather rely on governmental support and policy-based movement than work hard independently. The backward ideas and thoughts lead to "gap" for the transfer of farming people and boundaries as well as hinder population transfer and integration of urban and rural areas.

Restrictions of original system: First, household registration management. The traditional dual household registration system causes the unequal treatment between the farming people in cities and native urban residents. They cannot get access to social securities specific to urban residents ${ }^{[2]}$. Second, land management. The imperfection of land turnover system results in the non-standardization of land transfer procedure and market as well as the phenomenon of farmers' entry into cities with land because they only flow into cities without moving to cities. In addition, the industries develop in a backward manner. No adequate employment is available, which is also one reason for the difficulty in transfer of farming population.

Incomplete agricultural industry mechanism and imperfect economic \& social environment

Economic and social environment is not perfect. A sound economic and social environment is of critical importance for the development of agricultural industrialization. However, the current environment is not satisfying in many aspects ${ }^{[3]}$. If the government's functions cannot be transferred in time, the government should focus on the benefits of enterprises and farmers as well as plays the role of coordination in the agricultural industrialization. However, the government often intervenes with the specific execution too much and provides insufficient services. What's more, some governmental organizations and farmers cannot grasp information flexibly. They do not conform to market principles and cannot judge the plans of agricultural products $^{[3]}$. On the opposite, they are blind to making money. The industrialization is often low-end with few matching facilities and in lack of potentials. It cannot develop in a scientific manner. 
Single fund-raising channel in governmental department and incomplete investment \& financing system in rural and urban areas

The government's fund-raising channels are single. The fund for the construction of urbanization mainly comes from financial subsidiaries and specific funding. On the rural market, the investment $\&$ financing system is not perfect ${ }^{[5]}$. The policies are incomplete. Oriented by the market, the fund in the rural financial organizations is not input into rural construction. Instead, a majority flows onto the market for other commercial uses. Due to the single financial organizations in rural areas, local commercial banks and banks in towns do not give their roles into exertion. In addition, in terms of attracting social capital, there is a lack of valid mechanism. The threshold of inputting civil capital into the construction of public fields is so high that enterprises and individuals launch investment. The idle social fund cannot be allocated to the construction of urbanization. Furthermore, there are only a small number of financial branches in rural areas. The supply of fund is also in a small amount. Coupled with few financial service products, it is hard to attract more funds.

\section{Countermeasures of facilitating rural and urban integration}

Enhance publicity and ideological education

Strengthen cadres' ideological education

Through organizing cadres' learning, taking part in training, field visiting and many other forms, we can consolidate cadres' understanding of rural and urban integration, transform their wrong ideas, re-inspect integration policies, intensify their understanding of the profound connotations of urban and rural integration, combine the current situation and future, coordinate the development of economy, culture, society and environment, actually implement rational development into urban prosperity and construction, and facilitate urban and rural integration.

Enhance publicity among farmers

By means of innovative publicity, visiting, interview, propaganda columns, TV news and so on, the necessity and benefits of urban and rural integration should be publicized to every household. In particular, the policies and specific actions relevant to farmers' benefits in urban and rural integration as well as the vision into future production should be explained to farmers so that they can deepen the understanding of urban and rural integration and policies, transfer their ideas and combine themselves with urban and rural integration policies. In this way, the policies can be executed and farmers' life can be improved.

Enhance system reform

Enhance household registration reform

Household registration reform is a critical problem that must be solved in urbanization. It does not merely mean the transformation of identity, but also lies in the treatment after transformation, i.e., getting access to medical services, retirement pension, unemployment and other relevant social security policies and public services like the urban residents. The strategy of ladder-style facilitation can be adopted. The settlement conditions can then be loosened gradually. In addition, external immigrants' admission mechanism should be explored into. Besides, the reform should adapt to the requirements of industrial upgrading. Farmers who have a stable job in cities and the outsiders who possess certain technical titles, high professional level and academic background are encouraged to settle down. The central town belt and four major towns are highly urbanized. They can serve as the leading zones of household registration reform. As the pioneers that are transformed from farming land to non-farming land, they take the initiatives to realize the urbanization of towns in the middle part. Residents in remote towns are encouraged to gather in major towns. According to their possession of house property, jobs and term of residence in the cities, corresponding household transformation is implemented.

Facilitate the reform of social security system

The urban and rural social retirement insurance mechanism should be fully executed. The basic social retirement insurance system for employees in enterprises should be perfected. In addition, the coverage of 
residents' social retirement insurance in rural and urban areas should be expanded. In addition, the overall coverage of retirement insurance for rural residents should be realized as soon as possible. Farmers' retirement insurance should be improved gradually. The mechanism of normal adjustment of retired personnel's pension that is corresponding to the economic development in the province and city should be established. In the enterprises that have the ability, annual fund system should be set up to realize socialization management of retired personnel comprehensively. The basic pension insurance coverage should be expanded. The farmers' retirement insurance relationship should be flexibly extended. A uniform basic medical insurance system can be set up. The basic medical insurance system for rural and urban residents should be fully executed. The standard and level of medical subsidies in rural areas should be improved to gradually complete the connection of new rural medical insurance system with basic social medical security system. The basic medical insurance system for rural employees should be perfected. The coverage should be expanded to gradually improve the security level.

Perfect employment service system

Coordinate and formulate urban and rural employment planning

Rural and urban labor employment is included into the plan of economic and social development. The indicators of transfer employment and skill training should be adopted to evaluate the leaders. What's more, the plan of rural labor development employment should be drafted. The urban and rural boundary should be broken to create the environment where rural and urban employees can compete for jobs equally with knowledge, academic degree and skills. Furthermore, the system of unemployment registration, dynamic management and employment registration that integrates rural and urban areas should be set up. Public posts should be provided for those who have difficulty in seeking jobs. The surplus laborers in the residential zones should be included into the registration of urban employment and unemployment management.

Enhance quality cultivation-based quality of surplus rural laborers

The center of skill training for rural laborers should be set up. The labor training organizations with distinctive local features should be supported. According to the working needs, surplus laborers should be provided with skill training pertinently. Gradually, skill training and "industrialization" of recommended posts should be gradually formed so that the laborers who have received training can have the employment opportunity. In addition, skill training should be expanded to rural areas so as to facilitate the turnover of land contracting right. The moderate scale operation of land can be actually realized.

Accelerate the mechanism of agricultural industrialization

Promote the moderate concentration and scale operation of land

Encourage the transfer of land by means of outsourcing, transferring, share participation, cooperation, leasing, exchanging and so on within the term of land contracting. Encourage farmers to transfer the contracted land to large professional households, rural cooperation agency and agricultural park to develop large-scale agricultural operation. On the basis of setting up land turnover market, further standardize land transfer procedure and formulate a uniform land transfer convention for the purpose of ensuring the legal rights of farming households that transfer in and out the land. The subject that adheres to land transfer is farming household. The market and households decide the form, price and term of land transfer. According to laws, accelerate the system of verifying agricultural operation ability, classify, monitor and check the industrial and commercial enterprises, large farming households and other subjects. Accelerate the system of agricultural insurance, include the agricultural products within the scope of local land operation into the insurance scale. Explore into and set up land transfer risk control mechanism, perfect the system of land transfer guarantee, and reduce the risk of large-scale land transfer.

Set up industrial interaction development mechanism in rural and urban areas

Further adjust and optimize the industrial layout in urban and rural areas, clarify the orientation of industrial development. Through coordinating industrial planning, integrate industrial resources, perfect industrial system, and promote the integration and development of urban and rural industries. Accelerate the construction of 
relevant matching facilities that undertake industrial transfer in rural areas to promote the optimization and upgrading of industries in rural areas.

Enhance government's fund-raising and innovate investment \& financing system in urban and rural areas.

Strengthen guidance and governmental fund-raising

Continue to give the guiding role of governmental input into play, gradually increase a certain amount of fund for the planning and construction of urban and rural integration, and stimulate the initiatives of inputting social investment into urban construction. Increase the channels of government's fund-raising and apply government's fund as one source of the capital for rural construction. Give full play to the role of social fund. By means of risk undertaking, benefit sharing mechanism and preferential policy \& compensation provided by government for fund investors, attract social fund into the fund projects. Innovate the mode of fund operation. By means of investment union's funding mode, company's investment funding mode, limited partners' investment funding mode and so on, the effective operation mechanism of governmental funding can be formed. The use of governmental funding can be increased. Set up the loan risk compensation funding system to reduce the financing risks in medium and small enterprises as well as improve their financing ability.

Develop rural financial service system

On the prerequisite of giving full play to the functions of existing financial organizations, introduce the competition mechanism and try to construct a diverse new rural financial organization system. Give play to the backbone role of agricultural banks in supporting agriculture and rural economic development, try to expand each financial organization's right of approving of agricultural loans and reduce the threshold of credit loans. Deepen and perfect the reform of rural cooperation \& financial property system, improve the structure of financial legal person's governance and operation mechanism in rural areas, give play to the roles of rural agencies in the development of "three agriculture". Expand the scope of post savings bank's support for agriculture, guide and standardize the civil financial activities, encourage civil loan organizations that have a large scale, standard management and qualifications to transfer to new rural financial organizations. Actively develop rural banks, accelerate the facilitation of the pilot companies with a small amount of loans, and further expand the rural areas' financing channels.

\section{References}

[1] Shen Kongzhong, Transformation of Rural Communities that Integrate Urban and Rural Areas \& Coordinated Rural and Urban Development J. Human Geography.4(1999) 38-41.

[2] Zeng Wanming, Guide the Construction of Urban and Rural Integration with Scientific Outlook for Development J. Rural Economy.10 (2005) 25

[3] Wang Bifeng, Overview of Problems of Urban and Rural Integration J. Economic Theory and Business Management. 1(2004) 76

[4] Zhai Jiling, A Study on Economic Integration in Urban and Rural Areas and Countermeasures J. China Economic \& Trade Herald.18(2010):72.

[5] Chen Wen, A Discussion on Connotation of Urban and Rural Integration J. Modern Economic Research. $5(2003) 17$

[6] Li Cheng, Implications of Foreign Urban and Rural Integration on China J. Sci-tech Consultation, 21 (2005) 24 